[Radiocarbon, Vol 24, No. 1, 1982, P 45-53]

\title{
BIRBAL SAHNI INSTITUTE RADIOCARBON MEASUREMENTS III
}

G RAJAGOPALAN, VISHNU-MITTRE, B SEKAR, and T K MANDAL

Radiocarbon Laboratory, Birbal Sahni Institute of Palaeobotany Lucknow-226 007, India

The radiocarbon dates reported in this date list cover the measurements carried out in 1979 to 1980 . Chemical and counting procedures are the same as reported earlier (R, 1978, v 20, p 398-404). Age calculations are based on the conventional ${ }^{14} \mathrm{C}$ half-life $(5570 \mathrm{yr})$ and on the contemporary value of $95 \%$ of the activity of NBS oxalic acid. Errors quoted correspond to $l \sigma$ value which takes into account the counting statistics, the uncertainty in the half-life, and the instability of the counting system. The ages are not corrected for isotopic fractionation in nature.

Some modifications and improvements have been carried out in the electronics unit. A four-channel preset timer-printer unit has been incorporated with the scalers to record the counting data at preset time intervals. Statistical analyses of the printer output are regularly carried out for the detection of any abnormal behavior in the counting system and rejection of data using the methods described by Erlenkeuser and Willkomm (1972).

\section{ACKNOWLEDGMENTS}

Laboratory and technical help rendered by Prem Prakash, V S Panwar, and P S Salujha is thankfully acknowledged. The authors thank D K Biswas of Premier Electronics Ltd, Lucknow for help in the installation and testing of the timer-printer unit.

\section{Naini Tal series}

QUATERNARY SAMPLES

Peat and laminated clay samples from Naukuchiya Tal $\left(29^{\circ} 19^{\prime} \mathrm{N}\right.$, $79^{\circ} 35^{\prime}$ E) dist Naini Tal. Samples coll by boring, subm by H P Gupta, Birbal Sahni Inst Palaeobotany (BSIP) to date pollen diagrams.

Profile I (lake margin)

BS-116. Organic mud

Depth, 190 to $220 \mathrm{~cm}$ from swamp surface.

BS-114. Brownish gray peat

Depth, 290 to $320 \mathrm{~cm}$.

BS-115. Black humified peat

$4620 \pm 110$

Depth, 390 to $420 \mathrm{~cm}$.

BS-105. Peat with wood fragments

$7570 \pm 140$

Depth, 490 to $520 \mathrm{~cm}$.

BS-104. Humified clayey peat

$5240 \pm 120$

Depth, 590 to $620 \mathrm{~cm}$. 
Comment: pollen diagram of this profile is under preparation. Age of profile of pub pollen diagram from this lake (Vishnu Mittre, Gupta, and Robert, 1967; Gupta, 1977) was earlier interpreted much younger than these dates indicate.

Profile II (lake margin)

BS-140. Peat $3790 \pm 110$

Depth, 120 to $150 \mathrm{~cm}$.

BS-141.

$$
4340 \pm 120
$$

Clay with organic debris and wood fragments at depth 220 to $250 \mathrm{~cm}$.

\section{BS-142.}

$16,000 \pm 230$

Clay with wood fragments at depth 320 to $350 \mathrm{~cm}$.

Comment: in absence of hiatus in stratigraphy, Profile II indicates low sedimentation rate for compact portion of deposit. Pollen analytic results are awaited for comparison of pollen diagrams of Profiles I and II.

Terrace sediment from natural exposure, Bhim Tal lake $\left(29^{\circ} 24^{\prime} \mathrm{N}\right.$, $79^{\circ} 26^{\prime} \mathrm{E}$ ) dist Naini Tal. Coll and subm by H P Gupta to date terrace.

\section{BS-144. Sandy clay}

$620 \pm 100$

Depth, 140 to $160 \mathrm{~cm}$.

Terrace sediment from natural exposure, bore core profile, Ram Tal lake $\left(29^{\circ} 22^{\prime} \mathrm{N}, 79^{\circ} 32^{\prime} \mathrm{E}\right)$ dist Naini Tal. Coll and subm by H P Gupta to date climatic oscillations and development of lake basin.

BS-157. Clay

$120 \pm 130$

Depth, 45 to $60 \mathrm{~cm}$.

BS-158. Organic mul

$$
\mathbf{8 2 0} \pm \mathbf{8 0}
$$

Depth, 60 to $90 \mathrm{~cm}$.

BS-159. Brown peat

$940 \pm 70$

Depth, 110 to $140 \mathrm{~cm}$.

BS-160. Peat

$1400 \pm 100$

Depth, 190 to $215 \mathrm{~cm}$.

BS-161. Peat

$2410 \pm 100$

Depth, 235 to $265 \mathrm{~cm}$.

BS-162. Clay

$1550 \pm 70$

Depth, 385 to $415 \mathrm{~cm}$.

\section{Nilgiris series}

Peat and peaty clay samples from different swampy regions of dist Nilgiris. Samples coll by boring and subm by K Prasad, BSIP, to date pollen diagrams. 
Race course ( $\left.11^{\circ} 24^{\prime} 8^{\prime \prime} \mathrm{N}, 76^{\circ} 42^{\prime} \mathrm{E}\right)$.

BS-148. Peat

$1780 \pm 100$

Depth, 20 to $50 \mathrm{~cm}$.

BS-149. Peat

$1850 \pm 100$

Depth, 120 to $150 \mathrm{~cm}$.

BS-150. Peat

$2670 \pm 170$

Depth, 170 to $200 \mathrm{~cm}$.

Kappeathorai (11 $\left.21^{\prime} 50^{\prime \prime} \mathrm{N}, 76^{\circ} 38^{\prime} 40^{\prime \prime} \mathrm{E}\right)$.

BS-151. Peaty clay

Depth, 20 to $50 \mathrm{~cm}$.

Modern

Comment: other two samples of Kappeathorai profile at depths 70 to $100 \mathrm{~cm}$ and 120 to $150 \mathrm{~cm}$ were very low in carbon content, $i e$, undatable.

Kakathope $\left(11^{\circ} 35^{\prime} \mathrm{N}, 76^{\circ} 52^{\prime} \mathrm{E}\right)$.

BS-186. Peaty clay $2260 \pm 90$

Depth, 20 to $50 \mathrm{~cm}$.

BS-196. Peaty clay $5440 \pm 110$

Depth, 70 to $100 \mathrm{~cm}$.

BS-187. Peaty clay

Depth, 120 to $150 \mathrm{~cm}$.

$7530 \pm 130$

BS-197. Peaty clay

$10,310 \pm 170$

Depth, 170 to $200 \mathrm{~cm}$.

BS-188. Peaty clay

Depth, 220 to $250 \mathrm{~cm}$.

$14,060 \pm 220$

BS-198. Fibrous peaty clay

$21,600 \pm 470$

Depth, 270 to $300 \mathrm{~cm}$.

BS-199. Clay

$13,900 \pm 920$

Depth, 370 to $395 \mathrm{~cm}$.

Comment: dates support earlier estimate of sedimentation rate based on two ${ }^{14} \mathrm{C}$ dates (Agrawal, Gupta, and Kusumgar, 1969) for pollen diagram from this site. Pollen analytic studies of this profile are being made at present. Earlier pollen diagram revealed expansion of shrubs at $32,000 \mathrm{yr}$ BP into grassland savannah, formation of Shola forest ca 14,400 yr BP, its decline at 11,000 $\mathrm{yr}$ BP, and savannization later (Vishnu-Mittre, ms).

Colgrain $\left(11^{\circ} 35^{\prime} \mathrm{N}, 76^{\circ} 52^{\prime} \mathrm{E}\right)$.

BS-167. Peaty clay

$7360 \pm 120$

Depth, 20 to $50 \mathrm{~cm}$. 
BS-168. Peaty clay

$16,640 \pm 270$

Depth, 70 to $100 \mathrm{~cm}$.

BS-169. Clay

$11,820 \pm 160$

Depth, 120 to $150 \mathrm{~cm}$.

Comment: date of BS-167 agrees well with earlier measurement, BS-10, for another profile from this site (R, 1978, v 20, p 398-404).

\section{Rajasthan series}

Clay samples from trial trenches in Didwana lake $\left(27^{\circ} 30^{\prime} \mathrm{N}, 74^{\circ}\right.$ $30^{\prime} \mathrm{E}$ ) dist Nagaur to date climatic and vegetational history of Rajasthan. Coll and subm by A K Saxena, BSIP.

BS-123. Depth, 100 to $105 \mathrm{~cm}$

Modern

BS-121. Depth, 210 to $215 \mathrm{~cm}$

$5900 \pm 100$

Comment: date of BS-121 consistent with earlier measurements (R, 1978, v 20, p 398-404; R 1980, v 22, p 54-60). BS-123 is probably contaminated.

\section{Himachal Pradesh series}

Peat samples from lake margin of Parasram Tal, Nahan $\left(30^{\circ} 30^{\prime} \mathrm{N}\right.$, $70^{\circ} 28^{\prime}$ E) dist Sirmur. Samples coll by boring and subm by Chayya Sharma, BSIP, to date pollen diagram. Pollen analysis of profile is in progress.

BS-170. Depth, 100 to $130 \mathrm{~cm}$

$$
960 \pm 100
$$

BS-171. Depth, 170 to $200 \mathrm{~cm}$

$2750 \pm 100$

BS-172. Depth, 320 to $350 \mathrm{~cm}$

$4000 \pm 120$

BS-173. Depth, 460 to $490 \mathrm{~cm}$

$3140 \pm 100$

Renuka lake, Nahan $\left(30^{\circ} 30^{\prime} \mathrm{N}, 70^{\circ} 27^{\prime} \mathrm{E}\right)$.

BS-166. Clay

Modern

Depth, 30 to $50 \mathrm{~cm}$.

\section{Tripura and Manipur series}

Samples from trial trenches in peat deposits coll and subm by $\mathbf{A}$ Bhattachatrya, BSIP, to date pollen diagram.

Bisalgarh $\left(22^{\circ} 56^{\prime} \mathrm{N}, 91^{\circ} 10^{\prime} \mathrm{E}\right)$ dist West Tripura.

BS-208. Peat, 2.25m

$1750 \pm 100$

BS-209. Peat, 2.9m

$2600 \pm 90$

BS-210. Wood, 2.9m

$6930 \pm 120$

Chandrapur $\left(22^{\circ} 56^{\prime} \mathrm{N}, 91^{\circ} 15^{\prime} \mathrm{E}\right)$ dist West Tripura.
BS-205. Peat, $1.9 \mathrm{~m}$
$1870 \pm 100$
BS-206. Peat, 2.75m
$2980 \pm 110$ 
BS-207. Wood, 2.75m

$2040 \pm 90$

Comment: dates of wood samples are inconsistent.

Samples from peat deposits. Coll and subm by S Chanda, Bose Research Inst, Calcutta, to date pollen diagrams.

Kalpanya $\left(25^{\circ} \mathrm{N}, 91^{\circ} \mathrm{E}\right)$ dist Tripura. Samples coll by boring.

$\begin{array}{llr}\text { BS-152. } & 90 \text { to } 105 \mathrm{~cm} & 2940 \pm 200 \\ \text { BS-189. } & 195 \text { to } 215 \mathrm{~cm} & \text { Modern } \\ \text { BS-153. } & 260 \text { to } 275 \mathrm{~cm} & \text { Modern }\end{array}$

Comment: BS-189 and -153 are probably contaminated.

Sekerkot $\left(24^{\circ} \mathrm{N}, 91^{\circ} \mathrm{E}\right)$ dist Tripura. Samples coll from trial trench.

BS-174. Peat, 30 to $40 \mathrm{~cm}$

$1930 \pm 120$

BS-156. Wood, $380 \mathrm{~cm}$

$3340 \pm 140$

Lamphelpet $\left(25^{\circ} \mathrm{N}, 93^{\circ} \mathrm{E}\right)$ dist Manipur. Sample coll from trial trench.

BS-194. Peat, 35 to $40 \mathrm{~cm}$

$7980 \pm 470$

Turclu $\left(25^{\circ} \mathrm{N}, 93^{\circ} \mathrm{E}\right)$ dist Tripura. Sample coll from natural exposure.

BS-175. Lignite

$>40,000$

Tinsukia Forest Bungalow $\left(27^{\circ} 30^{\prime}\right.$ N, $95^{\circ} 30^{\prime}$ E) dist Upper Assam.

BS-154. Peat, $2.10 \mathrm{~m}$

$12,210 \pm 340$

Matikhad $\left(27^{\circ} 12^{\prime} \mathrm{N}, 95^{\circ} 48^{\prime} \mathrm{E}\right)$ dist Upper Assam.

BS-155. Peat, 2.5m

$\mathbf{1 7 , 9 2 0} \pm \mathbf{5 7 0}$

Wood and peat samples from Kantalia $\left(22^{\circ} 30^{\prime} \mathrm{N}, 88^{\circ} 27^{\prime} \mathrm{E}\right)$ dist Howrah, coll during systematic excavation. Coll and subm by B B Mukherjee, Bose Research Inst, Calcutta, to date sedimentologic processes.

BS-190. Peat, 3.29m

$1400 \pm 100$

BS-191. Wood, 4.03m

$5610 \pm 110$

BS-192. Wood, $4.61 \mathrm{~m}$

$5580 \pm 130$

BS-164. Wood, 7.34m

$6540 \pm 120$

BS-165. Wood, 7.93m

$5000 \pm 120$

Comment (BBM): BS-192 and -165 are inconsistent.

GEOLOGIC SAMPLES

\section{BS-124. Manjrod, Maharashtra}

$200 \pm 90$

Charcoal from natural exposure $100 \mathrm{~m}$ downstream on right bank of Tapti in Manjrod $\left(21^{\circ} 13^{\prime} \mathrm{N}, 74^{\circ} 59^{\prime} \mathrm{E}\right)$ dist Dhule. 
BS-125. Tamasvadi, Maharashtra

$250 \pm 100$

Charcoal from natural exposure right of Panjhra $\mathrm{R}, 300 \mathrm{~m} \mathrm{~N}$ of Tamasvadi $\left(21^{\circ} 03^{\prime} \mathrm{N}, 74^{\circ} 51^{\prime} 30^{\prime \prime} \mathrm{E}\right)$ dist Dhule. Comment: samples coll and subm by G V Rao, Geol Survey India to date sedimentologic history of Tapti and its sub-basins.

BS-139. Katra, Jammu \& Kashmir $>40,000$

Carbonaceous clay from natural exposure depth, $3 \mathrm{~m}$ from Katra $\left(33^{\circ} 15^{\prime} \mathrm{N}, 75^{\circ} 40^{\prime} \mathrm{E}\right)$, dist Udhampur. Coll to date Riasi thrust. Coll and subm by K S Krishnamurthy, Geol Survey India.

Peaty clay from trial trenches in area that formed Lohtak lake bed before canal excavation $\left(24^{\circ} 35^{\prime} 15^{\prime \prime} \mathrm{N}, 93^{\circ} 43^{\prime} \mathrm{E}\right)$, dist South Manipur. Coll and subm by Eng Geol Div, Geol Survey of India, to date geomorphologic and tectonic history of Lohtak basin under Loktak Hydel project.
BS-145. Peaty clay, depth $\mathbf{5 . 3 m}$
$11,470 \pm 190$
BS-147. Peaty clay, depth $12 \mathrm{~m}$
$25,500 \pm 660$

Peaty clay samples from dist Sirmur, Himachal Pradesh in black zone along Krol thrust. Coll and subm by Eng Geol Div, Geol Survey India, to date neotectonic activity along Krol thrust zone in Himalaya.

$\begin{array}{llr}\text { BS-183. } & \left(30^{\circ} 33^{\prime} \mathrm{N}, 77^{\circ} 48^{\prime} \mathrm{E}\right) & 31,000 \pm 1640 \\ \text { BS-184. } & \left(30^{\circ} 33^{\prime} \mathrm{N}, 77^{\circ} 42^{\prime} \mathrm{E}\right) & >40,000\end{array}$

Wood and peat samples coll and subm by S Subramaniam, Geol Survey India, to study development of coastal plain and neotectonic activity.

Vyasarpadi (13० $\left.7^{\prime} \mathrm{N}, 15^{\prime} 45^{\prime \prime} \mathrm{E}\right)$, dist Madras.

BS-185. Wood, depth 6.3 to $6.6 \mathrm{~m}$

$7000 \pm 120$

Sengammal $\left(12^{\circ} 45^{\prime} 50^{\prime \prime} \mathrm{N}, 80^{\circ} 12^{\prime} 50^{\prime \prime} \mathrm{E}\right)$, dist Chengalpat.

BS-193. Peat, depth 120 to $150 \mathrm{~cm}$

$6230 \pm 120$

BS-195. Carbonaceous sediment

$6400 \pm 160$

Depth, 120 to $150 \mathrm{~cm}$.

\section{BS-200. Gaik, Jammu \& Kashmir}

$6520 \pm 120$

Charcoal, depth $30 \mathrm{~cm}$ from natural exposure from Gaik $\left(35^{\circ} 20^{\prime} \mathrm{N}\right.$, $79^{\circ}$ E) dist Leh. Coll and subm by K K Sharma, Wadia Inst Himalayan Geol, Dehra Dun, to date terrace formation.

Shell samples from $\mathrm{E}$ coast of $\mathrm{S}$ India to date fossil sand dunes and eustatic changes. Samples coll and subm by R V Joshi, Deccan Coll, Pune. Idindakarai $\left(8^{\circ} 10^{\prime} \mathrm{N}, 77^{\circ} 45^{\prime} \mathrm{E}\right)$, dist Tirunelveli.

BS-134. Land snails, surface $20,000 \pm 400$ 
Kanyakumari $\left(8^{\circ} 2^{\prime} \mathrm{N}, 77^{\circ} 35^{\prime} \mathrm{E}\right)$, dist Kanyakumari.

BS-132. Marine shells, depth 2m

ARCHAEOLOGIC SAMPLES

\section{BS-43. Nirgudsar, Maharashtra}

$39,000 \pm 3200$

Wood from trench $6 \mathrm{~m}$ below modern bed level in cemented pebbly gravel, Nirgudsar ( $\left.18^{\circ} 31^{\prime} 30^{\prime \prime} \mathrm{N}, 74^{\circ} 22^{\prime} 30^{\prime \prime} \mathrm{E}\right)$ dist Pune. Sample to date Late Stone age culture of Ghod Valley. Subm by S N Rajguru, Deccan Coll, Pune.

\section{BS-146. Inamgaon, Maharashtra}

Shells at depth $90 \mathrm{~cm}$, from systematic excavation in Ghod valley of Late Stone age culture at Inamgaon (19 $\left.36^{\prime} 12^{\prime \prime} \mathrm{N}, 74^{\circ} 37^{\prime} 54^{\prime \prime} \mathrm{E}\right)$, dist Ahmednagar. Subm by S N Rajguru. Comment (SNR): dates agree with estimates based on geomorphic and lithostratigraphic history of Ghod valley.

\section{BS-163. Nandur Madmeshwar, Maharashtra $\quad \mathbf{2 6 , 6 0 0} \pm 430$}

Well-preserved shells at depth 7.62 to $9.14 \mathrm{~m}$ from natural exposure at Nandur Madmeshwar $\left(20^{\circ} \mathrm{N}, 74^{\circ} 5^{\prime} \mathrm{E}\right)$. Coll to date Middle Palaeolithic culture of upper Godawari valley. Comment (SNR): date agrees with estimates based on geomorphologic and palaeontologic correlations. Subm by $\mathrm{S} N$ Rajguru.

\section{BS-135. Peddarajupalli}

$1010 \pm 100$

Freshwater molluscan shells from natural exposure below red sandy loam layer, depth 10 to $20 \mathrm{~cm}$ found in assoc with artifacts of Upper Palaeolithic culture at Peddarajupalli $\left(14^{\circ} 4^{\prime} 30^{\prime \prime} \mathrm{N}, 79^{\circ} 22^{\prime} \mathrm{E}\right)$, dist Cuddapah. Subm by M L K Murthy, Deccan Coll, Pune. Comment: sample probably contaminated due to percolation of water from surface.

\section{BS-127. Dhupdhara, Assam}

$3370 \pm 130$

Wood from trial trench at depth $8 \mathrm{~m}$, from Dhupdhara $\left(25^{\circ} 26^{\prime} \mathrm{N}\right.$, $90^{\circ} 30^{\prime} \mathrm{E}$ ), dist Goalpara. Subm by M C Goswami, Dept Anthropol, Gauhati Univ, Gauhati.

\section{Daimabad series}

Charcoal samples in sequence from Jorwe to Late Harappa cultures from systematic excavation at Daimabad $\left(1^{\circ} 30^{\prime} \mathrm{N}, 74^{\circ} 31^{\prime} \mathrm{E}\right)$, dist Ahmednagar. Coll and subm by S A Sali, Archaeol Survey India, Aurangabad.

BS-176. Trench GZ 63

$$
3590 \pm 90
$$

Sample from depth 4.1m, Sec I, Layer (15), House No. 12.

BS-177. Trench $Z^{\prime} 3$

$$
3460 \pm 100
$$

Sample from depth 2.25m, Sec II, Pit 145, sealed by (10A). 
BS-178. Trench Y'2

Sample from depth $10 \mathrm{~cm}$, Kiln No. 1, sealed by (1), Sec II.

BS-179. Trench $\mathrm{DZ}^{\prime} 3$

$2970 \pm 100$

Sample from depth $30 \mathrm{~cm}$, upper levels of House No. 38, Sec II.

BS-180. Trench ZD60

$3390 \pm 100$

Sample from depth $2.3 \mathrm{~m}$, Sec IV, from hearth sealed by (11).

BS-181. Trench $\mathbf{Y}^{\prime} \mathbf{3}$

$2990 \pm 100$

Sample from depth $1.36 \mathrm{~m}$, Sec II, Layer (7).

BS-182. Trench $\mathrm{Z}^{\prime} \mathbf{3}$

$3130 \pm 90$

Sample from depth $2.1 \mathrm{~m}$, Sec II, Layer (10).

Comment (SAS): dates for BS-176,-180,-181, and -182 are stratigraphically inconsistent. Dates also do not agree with estimates for various phases of Daimabad series based primarily on ${ }^{14} \mathrm{C}$ dates for Malwa and Jorwe cultures from Inamgaon, Chandoli, Songaon, and Navasa in Maharashtra and chronology of Harappa culture.

\section{Allahabad series}

Samples from various phases of Mesolithic and Upper Palaeolithic cultures in Ganga valley coll during systematic excavation. Subm by $G \mathrm{R}$ Sharma, Dept Ancient Hist \& Archaeol, Allahabad Univ.

Mahagara $\left(24^{\circ} 54^{\prime} 50^{\prime \prime} \mathrm{N}, 82^{\circ} 3^{\prime} 20^{\prime \prime} \mathrm{E}\right)$ dist Allahabad.

BS-130. Trench L/6

Shell from depth 17 to $33 \mathrm{~cm}$, upper level cemented gravel III, Loc XXX-XXXVI.

BS-131. Trench L/6

$9830 \pm 160$

Shell from depth 33 to $73 \mathrm{~cm}$, middle level cemented gravel III, Loc XXX-XXXVI.

BS-128. Trench G/7

$3330 \pm 100$

Charcoal from depth 2.8 to $3.5 \mathrm{~m}$, Pit 8B(18), Loc XXXVI-XXXVII.

Chopani Mando $\left(25^{\circ} 34^{\prime} \mathrm{N}, 81^{\circ} 53^{\prime} \mathrm{E}\right)$ dist Allahabad.

BS-129. Trench F/5

$4540 \pm 110$

Charcoal from depth 20 to $30 \mathrm{~cm}$, Layer (2A) and (3), Loc I-VI.

Mahadaha (25 $\left.59^{\prime} 2^{\prime \prime} \mathrm{N}, 82^{\circ} 11^{\prime} 30^{\prime \prime} \mathrm{E}\right)$ dist Pratapgarh.

BS-136. Trench $F / 3$ (cemetery area)

$4010 \pm 120$

Charred bones (carbonate) from depth 20m, Pit 8B(2), Loc XII-XIV.

BS-137. Trench $\mathbf{F} / 3$

$\mathbf{2 8 8 0} \pm \mathbf{2 5 0}$ XII-XVIII.

Charred bones (carbonate) from depth 16 to $26 \mathrm{~cm}$, Layer (3), Loc 
BS-138. Trench $F / 2$

$\mathbf{3 8 4 0} \pm \mathbf{1 3 0}$ VI-XII.

Charred bones (carbonate) from depth 27 to $42 \mathrm{~cm}$, Layer (4), Loc

Comment (GRS): dates are much younger than estimates based on PRL-100 and -101 (Agrawal et al, 1976). Samples are probably contaminated by percolation of water through secs.

\section{Satanikota series}

Charcoal samples from Satanikota $\left(15^{\circ} 56^{\prime} \mathrm{N}, 78^{\circ} 14^{\prime} \mathrm{E}\right)$, dist Kurnool, coll during systematic excavation of megalithic site. Subm by N C Ghosh, Archaeol Survey India, Nagpur, to date megaliths of $S$ India.

$\begin{array}{lll}\text { BS-201. } & \text { Depth, } 0.75 \mathrm{~m} & 1620 \pm 100 \\ \text { BS-202. } & \text { Depth, } 1.7 \text { to } 1.85 \mathrm{~m} & 1440 \pm 100 \\ \text { BS-203. } & \text { Depth, } 1.95 \mathrm{~m} & 7520 \pm 140 \\ \text { BS-204. } & \text { Depth, } 2.55 \mathrm{~m} & 8960 \pm 120\end{array}$

Comment: BS-201 and -202 are inconsistent with estimate. BS-203 and -204 agree with estimates based on assoc Black and Red ware and red slipped sherds belonging to Megalithic period.

\section{REFERENCES}

Agrawal, D P, Gupta, S K, and Kusumgar, Sheela, 1969, Tata Institute radiocarbon date list VII: Radiocarbon, v 11, p 503-508.

Agrawal, D P, Krishnamurthy, R V, Kusumgar, Sheela, and Pant, R K, 1976, Physical Research Laboratory radiocarbon date list I: Radiocarbon, v 18, p 89-95.

Erlenkeuser, $H$ and $W$ illkomm, $\mathrm{H}, 1972$, Data processing in ${ }^{14} \mathrm{C}$ measurements: Atomkernenergie (ATKE), v 19, p 123-126.

Gupta, H P, 1977, Pollen analytical reconnaissance of post-glacial deposits from subtropical zone in Naini Tal Dist Kumaon Himalaya: Palaeobotanist, v 24, p 215-244.

Rajagopalan, G, Vishnu-Mittre, and Sekar, B, 1978, Birbal Sahni Institute radiocarbon measurements I: Radiocarbon, v 20, p 398-404. v 22, p 54-60.

Vishnu-Mittre, ms, Biostratigraphy of Kakathope pollen profile from Ootacamund, Nilgiris, India, in press.

Vishnu-Mittre, Gupta, H P, and Robert, R D, 1967, Studies of the late Quaternary vegetational history of Kumaon Himalaya: Current Sci, v 36, p 539-540. 